\title{
Effectiveness of the 2011-12 Influenza Vaccine: Data from US Military Dependents and US-Mexico Border Civilians
}

\author{
Damaris Padin*, Anthony Hawksworth, Peter Kammerer, Erin McDonough and Gary Brice \\ Operational Infectious Diseases, Naval Health Research Center, San Diego, CA, USA
}

\section{Objective}

To assess effectiveness of the influenza vaccine among US military dependents and US-Mexico Border populations during the 2011-12 influenza season.

\section{Introduction}

As a result of antigenic drift of the influenza viruses, the composition of the influenza vaccine is updated yearly to match circulating strains. Consequently, there is need to assess the effectiveness of the influenza vaccine (VE) on a yearly basis. Ongoing febrile respiratory illness (FRI) surveillance captures data and specimens that are leveraged to estimate influenza VE on an annual basis.

\section{Methods}

Data from ongoing FRI surveillance at US Military and US-Mexico border clinics were used to estimate VE. We conducted a casecontrol study between weeks 3 and 17 of the 2011-12 influenza season. Specimens were collected from individuals meeting FRI case definition (fever $\geq 100.0 \mathrm{~F}$ with either cough or sore throat). Cases were laboratory confirmed influenza infection and controls were negative for influenza. Interviewer-administered questionnaires collected information on patient demographics and clinical factors and vaccination status. Logistic regression was used to calculate the crude and adjusted odds ratios (OR) and VE was computed as (1-OR) x 100\%. Vaccine protection was assumed to begin 14 days post-vaccination.

\section{Results}

A total of 155 influenza positive cases and 429 influenza negative controls were included in the analysis - 72 cases were influenza $\mathrm{A}(\mathrm{H} 3 \mathrm{~N} 2), 38$ cases were influenza $\mathrm{A}(\mathrm{H} 1 \mathrm{~N} 1)$, and 45 cases were influenza B. Overall adjusted VE against laboratory-confirmed influenza was 46\% (95\% CI, 19-64\%); unadjusted was 39\% (95\% CI, 11-58\%). Influenza subtype analyses revealed moderate protection against $\mathrm{A} / \mathrm{H} 3$ and $\mathrm{A} / \mathrm{H} 1$ and lower protection against $\mathrm{B}$. Lowest estimated VE was seen in older individuals, age 65 and older.

\section{Conclusions}

Influenza vaccination was moderately protective against laboratory confirmed influenza in this population. Continued surveillance is important in monitoring the effectiveness of the influenza vaccine.

\section{Keywords}

Influenza; vaccine efficacy; Influenza-like illness surveillance

\section{Acknowledgments}

The authors gratefully acknowledge the work of on-site research assistants and NHRC laboratory staff who produced the data used in this analysis. We also thank Global Emerging Infections System (GEIS) division of the Armed Forces Health Surveillance Center (AFHSC) for their support of FRI surveillance.

*Damaris Padin

E-mail: damaris.padin@med.navy.mil 